\begin{tabular}{|c|l|}
\hline Title & Autophagy in the placenta of women with hy pertensive disorders in pregnancy \\
\hline Author(s) & $\begin{array}{l}\text { A kaishi, Rina; Yamada, Takahiro; Nakabay ashi, Kazuhiko; Nishihara, Hiroshi; Furuta, Itsuko; Kojima, Takashi; } \\
\text { Morikawa, Mamoru; Y amada, Takashi; Fujita, Naonobu; Minakami, Hisanori }\end{array}$ \\
\hline Citation & $\begin{array}{l}\text { Placenta, 35(12), 974.980 } \\
\text { https://doi.org/10.1016/.placenta.2014.10.009 }\end{array}$ \\
\hline Issue Date & 201412 \\
\hline Doc URL & http://hdl.handle.net/2115/57971 \\
\hline Type & article (author version) \\
\hline File Information & text.pdf \\
\hline
\end{tabular}

Instructions for use 


\section{Autophagy in the placenta of women with hypertensive disorders in pregnancy}

Rina Akaishi ${ }^{\mathrm{a}}$, Takahiro Yamada ${ }^{\mathrm{a} *}$, Kazuhiko Nakabayashi ${ }^{\mathrm{b}}$, Hiroshi Nishihara ${ }^{\mathrm{c}}$, Itsuko Furuta $^{a}$, Takashi Kojima ${ }^{a}$, Mamoru Morikawa ${ }^{a}$, Takashi Yamada ${ }^{a}$, Naonobu Fujita ${ }^{d}$, 5 Hisanori Minakami ${ }^{\mathrm{a}}$

Affiliations of all authors:

${ }^{a}$ Department of Obstetrics and Gynecology, Hokkaido University Graduate School of Medicine, Sapporo, 060-8638, Japan.

$10{ }^{\mathrm{b}}$ Department of Maternal-Fetal Biology, National Research Institute for Child Health, Tokyo, Japan

${ }^{c}$ Departments of Translational Pathology, Hokkaido University Graduate School of Medicine, Sapporo, Japan

${ }^{\mathrm{d}}$ Department of Genetics, Osaka University Graduate School of Medicine, Japan.

15 and Development, Suita, Japan

*Correspondence to: Takahiro Yamada,

Department of Obstetrics and Gynecology, Hokkaido University Graduate School of Medicine, N15W7, Kita-ku, Sapporo, 060-8638, Japan. TEL:+81-11-706-5941,

20 FAX:+81-11-706-7711.

E-mail address: taka0197@med.hokudai.ac.jp 
Akaishi R et al.,

Short running title:

Autophagy in the human placenta in hypertensive disorders 


\begin{abstract}
Introduction: Autophagy has not been studied extensively in the human placenta. This study was performed to determine whether autophagy is increased in the placentas of women with hypertensive disorders in pregnancy compared to normotensive pregnancies.
\end{abstract}

Methods: LC3-II and p62 protein expression were examined by quantitative Western blotting analysis in 40 placentas from women not experiencing labor pains. The 40 placentas were from 13, 8, and 19 women with preeclampsia, gestational hypertension, and normal pregnancy, respectively. Hypertensive disorders in pregnancy included preeclampsia and gestational hypertension.

Results: LC3-II expression was significantly increased, while that of p62 was significantly reduced in 21 placentas of women with hypertensive disorders compared to those with normal blood pressure irrespective of the presence or absence of fetal growth restriction (FGR). LC3-II expression was also significantly increased in 13 placentas of women with preeclampsia irrespective of the presence or absence of FGR.

Discussion: The results of this study suggested that autophagy is active in the placenta of hypertensive disorders even in the absence of FGR.

Keywords: Autophagy, gestational hypertension, preeclampsia, pregnancy-induced hypertension 


\section{Autophagy in the placenta of women with hypertensive disorders in pregnancy}

Rina Akaishi ${ }^{\mathrm{a}}$, Takahiro Yamada ${ }^{\mathrm{a} *}$, Kazuhiko Nakabayashi ${ }^{\mathrm{b}}$, Hiroshi Nishihara ${ }^{\mathrm{c}}$, Itsuko Furuta $^{a}$, Takashi Kojima ${ }^{a}$, Mamoru Morikawa ${ }^{a}$, Takashi Yamada ${ }^{a}$, Naonobu Fujita ${ }^{d}$, 5 Hisanori Minakami ${ }^{\mathrm{a}}$

Affiliations of all authors:

${ }^{a}$ Department of Obstetrics and Gynecology, Hokkaido University Graduate School of Medicine, Sapporo, 060-8638, Japan.

$10{ }^{\mathrm{b}}$ Department of Maternal-Fetal Biology, National Research Institute for Child Health, Tokyo, Japan

${ }^{c}$ Departments of Translational Pathology, Hokkaido University Graduate School of Medicine, Sapporo, Japan

${ }^{\mathrm{d}}$ Department of Genetics, Osaka University Graduate School of Medicine, Japan.

15 and Development, Suita, Japan

*Correspondence to: Takahiro Yamada,

Department of Obstetrics and Gynecology, Hokkaido University Graduate School of Medicine, N15W7, Kita-ku, Sapporo, 060-8638, Japan. TEL:+81-11-706-5941,

20 FAX:+81-11-706-7711.

E-mail address: taka0197@med.hokudai.ac.jp 
Akaishi R et al.,

Short running title:

25 Autophagy in the human placenta in hypertensive disorders 


\begin{abstract}
Introduction: Autophagy has not been studied extensively in the human placenta. This study was performed to determine whether autophagy is increased in the placentas of women with hypertensive disorders in pregnancy compared to normotensive pregnancies.

Methods: LC3-II and p62 protein expression were examined by quantitative Western blotting analysis in 40 placentas from women not experiencing labor pains. The 40 placentas were from 13, 8, and 19 women with preeclampsia, gestational hypertension, 35 and normal pregnancy, respectively. Hypertensive disorders in pregnancy included preeclampsia and gestational hypertension.

Results: LC3-II expression was significantly increased, while that of p62 was significantly reduced in 21 placentas of women with hypertensive disorders compared to those with normal blood pressure irrespective of the presence or absence of fetal

40 growth restriction (FGR). LC3-II expression was also significantly increased in 13 placentas of women with preeclampsia irrespective of the presence or absence of FGR.

Discussion: The results of this study suggested that autophagy is active in the placenta of hypertensive disorders even in the absence of FGR.
\end{abstract}

45 Keywords: Autophagy, gestational hypertension, preeclampsia, pregnancy-induced hypertension 
Akaishi R et al.,

Abbreviations:

PIH: pregnancy-induced hypertension

50 FGR: fetal growth restriction

PE: preeclampsia

$\mathrm{GH}$ : gestational hypertension

SBP: systolic blood pressure

DBP: diastolic blood pressure

55 GW: gestational week 


\section{Introduction}

Autophagy is an intracellular bulk degradation system used to recycle cytoplasmic components [1]. As autophagy is activated under conditions of nutritional deprivation and intracellular stress, such as hypoxia, it is thought to be pivotal for the cellular response to starvation and stress as well as the normal turnover of cytoplasmic constituents [2].

As autophagy has only recently been identified in the human placenta [3], to our knowledge, there have been only eight reports dealing with autophagy in the human

65 placenta $[3,4,5,6,7,8,9,10]$. Two of these reports dealt with placentas of women with preeclampsia (PE) [3, 5]. However, the results were inconsistent: one suggested increased autophagy in PE [3], while the other did not [5]. Three reports dealt with placentas in cases of fetal growth restriction (FGR) $[5,7,8]$, and all three suggested increased autophagy in placentas with FGR.

70 The activation of autophagy is reflected by increases in the abundance of key proteins of the autophagy-related pathways, such as beclin-1 and microtubule-associated protein 1A/1B-light chain 3 (LC3) [11]. LC3 is distributed ubiquitously in mammalian tissues, the cytosolic form of LC3, LC3-I, is converted to LC3-II and recruited to autophagosomal membranes during autophagy, LC3 is the most widely monitored autophagy-related protein and LC3-II is the only protein marker that is reliably associated with completed autophagosomes [11]. Thus, detecting LC3-II by immunoblotting or immunofluorescence is a reliable means of monitoring autophagy $[11,12]$. As p62 protein, also called sequestosome 1 , is itself degraded by autophagy and accumulates when autophagy is inhibited, p62 can also be used as a marker to study autophagic flux $[11,13]$. Thus, the coexistence of both increased LC3-II expression and decreased p62 protein may be seen simultaneously in placentas with increased autophagic activity. Here, we report the results of a study addressing whether autophagy monitored by both LC3-II and p62 is increased in the placentas of women with hypertensive disorders in pregnancy compared with those with normotensive 85 pregnancies.

\section{Materials and Methods}

This study was conducted with the approval of the Ethics Committee of Hokkaido University Graduate School of Medicine and written informed consent was obtained from each participant.

\section{Source of placentas}

A total of 40 placentas from 40 women with singleton pregnancies were examined in this study (Table 1); 21 placentas from 21 women with pregnancy-induced hypertension (PIH) consisting of 13 women with PE and 8 women with gestational hypertension (GH) and 19 placentas from 19 women without PIH. All of the 40 women gave birth by elective caesarean section at our hospital during the 3.5-year study period between October 2005 and March 2009. Women with multiple pregnancies, chronic 
hypertension, and chorioamnionitis were excluded from the present study. Women with emergency caesarean section after onset of labor were excluded from the present study because autophagy status is altered by labor pains [4].

100 Gestational hypertension $(\mathrm{GH})$ was defined as hypertension (systolic blood pressure $\geq$ $140 \mathrm{mmHg}$ and/or diastolic blood pressure $\geq 90 \mathrm{mmHg}$ on at least two occasions recorded more than $12 \mathrm{~h}$ apart) occurring on and after gestational week (GW) 20 without proteinuria. The proteinuria was defined as a protein-to-creatinine ratio $(\mathrm{mg} / \mathrm{mg}) \geq 0.27$ in spot urine test or protein loss in urine $>0.3 \mathrm{~g} /$ day occurring on or

105 after GW 20. A diagnosis of PE was made when women developed both GH and proteinuria. FGR was defined as a birth weight less than $10^{\text {th }}$ percentile for Japanese infants for each GW [14].

\section{Preparation of placental specimens}

Placental specimens (approximately $1.0 \mathrm{~g}$ ) obtained from the central mid-portion

110 between the basal plate and chorionic membrane mainly containing the villous tissue were washed with normal saline and stored at $-80^{\circ} \mathrm{C}$ until use for protein extraction.

\section{Western blotting analysis for detection of LC3-II and p62}

Proteins were extracted after mixing of $20 \mathrm{mg}$ villous tissue with $100 \mu \mathrm{L}$ of $2 \%$ Triton X-100 lysis buffer (20 mM Tris-HCl, $150 \mathrm{mM} \mathrm{NaCl}, 2 \%$ Triton X-100, 2 mM PMSF, 115 and Complete Protease Inhibitor Cocktail), sonication, and centrifugation $(16100 \times g$, $\left.10 \mathrm{~min}, 4^{\circ} \mathrm{C}\right)$. Protein concentration in the supernatant was determined by Pierce BCA Protein Assay (Thermo Fisher Scientific, Rockford, IL). The protein samples (15 $\mu \mathrm{g})$ were diluted with 1:2 volumes $(\mathrm{mL} / \mathrm{mL})$ of Laemmli sample buffer $(10 \% \mathrm{SDS}, 1 \mathrm{M}$ DTT, $0.5 \mathrm{M}$ Tris-HCl, pH 6.8, 0.001\% bromophenol blue, and 5\% glycerol), boiled at

$12095^{\circ} \mathrm{C}$, separated by SDS-polyacrylamide gel electrophoresis (13\% for LC3 and $8 \%$ for p62), and then electrotransferred onto PVDF membranes (Amersham Biosciences, Little Chalfont, UK). After blocking with $1.5 \%$ skim milk in TBS-T $(1 \times$ TBS with $0.08 \%$ Tween 20) at room temperature for $1 \mathrm{~h}$, the membranes were incubated with primary antibody at $4{ }^{\circ} \mathrm{C}$ overnight. As a control, GAPDH was detected using an anti-GAPDH

125 mouse IgG antibody (1:107 dilution; Cat No.MAB374, Lot.LV1395312, Chemicon International Inc, Temecula, CA). LC3 was detected using a monoclonal anti-LC3 rabbit IgG antibody (1:1000 dilution; Cat No. PM036, Lot.010, MBL, Nagoya, Japan) and p62 was detected using polyclonal anti-p62/SQSTM1 rabbit IgG antibody (1:2000 dilution; Cat No. PM045, Lot.015, MBL, Nagoya, Japan). After washing with TBS-T,

130 the membranes were incubated with horseradish peroxidase-conjugated anti-mouse IgG secondary antibody (Amersham Biosciences) for GAPDH and horseradish peroxidase-conjugated anti-rabbit IgG secondary antibody (Amersham Biosciences) for LC3 and p62 at a dilution of 1:10000 at room temperature for $1 \mathrm{~h}$. After washing again with TBS-T, the membranes were transferred to ECL solution (Immobilon ${ }^{\mathrm{TM}}$ Western;

135 Amersham Biosciences) and the immunoreactive band intensities were quantified using an LAS-4000 mini chemiluminescence imaging system (Fuji Film, Tokyo, Japan). GAPDH was used as a loading control (Fig. 1). Median values for LC3-II and p62 protein expression were obtained in 40 placentas and each datum was transformed to a 
value of multiple of the median (MoM, absolute value divided by the median) before comparison.

\section{Immunohistochemistry and immunofluorescence study}

Immunohistochemical studies were carried out on $4 \mu \mathrm{m}$ thick tissue sections from formalin-fixed, paraffin-embedded tissue's samples, using a Novolink Polymer Detection System (Novocastra Laboratories) with primary mouse monoclonal anti-p62

145 antibody (M162-3, MBL, Nagoya, Japan), primary rabbit polyclonal anti-LC3 antibody (PM036,MBL, Nagoya, Japan). Sections were deparaffinized in Bio-Clear for 20 minutes then washed twice in ethanol. Antigen-retrieval bath containing $0.25 \mathrm{mM}$ EDTA at $\mathrm{pH} 9$ for 30 minutes at $95^{\circ} \mathrm{C}$ was used for p62 and LC3. Endogenous peroxidase activity was quenched with $3 \% \mathrm{H}_{2} \mathrm{O}_{2}$ in distilled $\mathrm{H}_{2} \mathrm{O}$. Staining was

150 performed with diaminobenzidine and fast red as a chromogen. For LC3 and p62 staining, the primary antibody was applied at the dilutions of 1:500 and 1:50, respectively and incubated overnight at $4^{\circ} \mathrm{C}$. Slides with absence of the primary antibody were included as negative controls. Slides were immunostained in the same batch to ensure identical condition for comparison. images were viewed and captured 155 using Olympus BX43 microscope (Olympus, Tokyo, Japan).

For immunofluorescence study, paraffin-embedded tissue sections were deparaffinized in xylene and rehydrated through a graded series of alcohols. P62 and LC3 were detected using primary mouse monoclonal anti-p62 antibody (M162-3, MBL, Nagoya, Japan), primary rabbit polyclonal anti-LC3 antibody (PM036,MBL, Nagoya, Japan),

160 respectively. Slides were incubated overnight at $4^{\circ} \mathrm{C}$. Fluorophore-conjugated secondary antibodies were employed as follows: Alexa Fluor ${ }^{\circledR} 594$ Donkey Anti-Mouse IgG $(\mathrm{H}+\mathrm{L}) \quad$ (A21203 Molecular Probes ${ }^{\circledR}$, Life Technologies, Carlsbad, CA) for p62 staining, Alexa Fluor ${ }^{\circledR} 488$ Donkey Anti-Rabbit IgG (H+L) (A-21206 Molecular Probes ${ }^{\circledR}$, Life Technologies, Carlsbad, CA) for LC3. In the negative controls,

165 the primary antibody was omitted and the specificity of the primary antibody was checked. Fluorescence images were viewed and captured using Olympus IX73 microscope (Olympus, Tokyo, Japan).

\section{Statistical analysis}

All data are presented as the means \pm SD or frequency. Fisher's exact test was used for

170 comparison of categorical data and Student's $t$ test or Mann-Whitney U-test was used to compare median values. In all analyses, $P<0.05$ was taken to indicate statistical significance. The relations between the results were investigated by Pearson's correlation coefficient analysis or Spearman's rank correlation analysis. All analyses were carried out using the SPSS Statistical Analysis System version 12 (SPSS Inc.,

175 Chicago, IL).

\section{Results}


The demographic characteristics of the participants are shown in Table 1. Compared with 19 control women without PIH, elective caesarean delivery occurred at a significantly earlier stage of pregnancy, fractions of women with preterm delivery

180 (gestational week <37) and FGR infants were significantly greater, and infant birth weight as well as z-score of birth weight were significantly smaller in the 21 women with PIH. All seven cases of FGR belonged to the PIH group

\section{Effects of gestational week and association of LC3-II and p62 protein expression}

No significant linear relationship was observed between the two variables of LC3-II and p62 protein expression (Fig. 1). There were 7 and 6 placentas with LC3-II level $>2.0$ and p62 levels $>2.0$, respectively. However, no placentas exhibited LC3-II level $>2.0$ and p62 levels $>2.0$. Thus, placentas of higher LC3-II levels appeared not to have higher p62 levels. In addition, correlation analyses suggested that the LC3-II level decreased with advancing GW at delivery $(P=0.024)$, while the p62 level tended to

190 increase with advancing GW at delivery $(P=0.072)$ in the 19 placentas of control women (Fig. 2, upper panel). These trends were not seen in the 21 placentas from women with PIH (Fig. 2, lower panel). Although the LC3-II and p62 levels did not differ significantly between the two groups divided by GW at delivery, possibly due to the limited size of the study population, LC3-II level was significantly higher and p62

195 level was significantly lower in the placentas of PIH women than in those of control women among placentas with advanced gestation (Tables 2, 3).

\section{Effects of hypertensive disorders in pregnancy on expressions of LC3-II and p62 protein}

According to MoM levels of LC3-II and p62, 40 placentas were grouped into four groups, including 9 placentas with LC3-II $>1.0$ and p62 $>1.0$, 11 placentas with LC3-II

$200>1.0$ and $\mathrm{p} 62<1.0,11$ placentas with LC3-II $<1.0$ and $\mathrm{p} 62>1.0$, and 9 placentas with LC3-II $<1.0$ and p62 $<1.0$ (Fig. 3). PIH occurred exclusively among women with placentas other than those with LC3-II $<1.0$ and p62 $>1.0(72 \%[21 / 29]$ vs. $0.0 \%$ [0/11], $P<0.0001)$. FGR occurred exclusively among women with placentas with LC3-II $>1.0(35 \%[7 / 20]$ vs. $0.0 \%[0 / 20], P=0.008)$. The frequency of PIH was

205 significantly higher in 20 women with placentas with LC3-II $>1.0$ than in 20 with LC3-II $<1.0(75 \%[15 / 20]$ vs. $30 \%$ [6/20], respectively, $P=0.010)$.

The degrees of LC3-II and p62 expression were analyzed according to the presence or absence of hypertension, proteinuria, and FGR. Compared with 19 control placentas without hypertension, proteinuria, or FGR, the expression of LC3-II was significantly

210 increased in all except one group and the expression of p62 was significantly reduced in placentas with PIH $(n=21)$ or PIH but without FGR $(n=14)$ (Fig. 4). Thus, PIH (hypertension with or without proteinuria) was associated with both increased LC3-II expression and reduced p62 expression irrespective of the presence or absence of FGR. Although LC3-II was significantly increased in 8 PE placentas without FGR, p62 was not significantly reduced compared to 19 normal pregnancies. 
Associations between normalized infant birth weight for gestational age (z-score) and LC3-II and p62 protein expression

Although not statistically significant, expression of LC3-II protein tended to decrease linearly with increasing z-score of infant birth weight $(P=0.087)$ (Fig. 5). No such tendency was observed in expression of $\mathrm{p} 62$ protein. The median z-score was -0.3 for the 40 placentas. Although the frequency of PIH did not differ significantly between the two groups divided by median z-score (65\% [13/20] for lower z-score group vs. $40 \%$ [8/20] for higher $\mathrm{z}$-score group, $P=0.2049)$, the expression of LC3-II protein was significantly greater in the lower z-score group than the higher z-score group $(1.45 \pm$

2250.82 vs. $0.93 \pm 0.71$, respectively, $P=0.044$ ), while the expression of p62 did not differ significantly between the two groups $(0.93 \pm 0.71$ vs. $1.42 \pm 1.51$, respectively, $P=$ $0.414)$.

\section{Localization of $L C 3$ and $p 62$}

Immunofluorescence method confirmed the co-localization of LC3 and p62 in villous

230 trophoblasts (Fig. 6A). Immunohistochemical staining showed that LC3 and p62 were localized in villous cyto- and syncytio-trophoblasts and extravillous trophoblast (Fig. $6 \mathrm{~B})$.

\section{Discussion}

The present study demonstrated that a significant increase in the expression of LC3-II

235 concomitant with a significant decrease in the expression of p62 occurred in women with PIH irrespective of the presence or absence of FGR. LC3-II was also significantly increased in the placentas of women with PE irrespective of the presence or absence of FGR. Analyses of changes in LC3-II and p62 levels according to GW at delivery suggested that autophagic activity decreased with advancing gestation in the placentas

240 of control women with normal blood pressure, consistent with the results reported previously [6], whereas this change did not occur in the placentas of PIH women. These observations suggested that active placental autophagy continued until term in women with hypertensive disorders even in the absence of FGR, but not in those with normal blood pressure.

245 There have been no previous reports regarding human placental expression of p62. Inhibition of autophagy is correlated with increased levels of p62 in mammals, suggesting that steady-state levels of this protein reflect the autophagic status. Similarly, decreased p62 levels are associated with the activation of autophagy [11,13]. Indeed, decreases in p62 expression were observed after activation of autophagy in the human

250 heart [15] and in a rat model of myocardial hypertrophy [16]. Therefore, the expression of p62 was expected not to be high in the placentas with increased LC3-II levels. Indeed, all 7 placentas with LC3-II level $>2.0$ exclusively showed p62 level $<2.0$ (Fig. 1). In addition, placentas of PIH with a significantly higher LC3-II level showed a significantly lower p62 level compared to GW-matched placentas of control women 255 (Tables 2, 3). Thus, the determination of p62 level in addition to LC3-II level may be useful for the assessment of autophagic status of the villous tissue. 
There were two previous reports regarding autophagy in the placenta of women complicated with hypertensive disorders in pregnancy [3,5]. The first report by Oh et al. [3], examining 19 placentas in women without labor pains, demonstrated that placental

260 LC3-II expression was increased in 11 women with severe PE compared to 8 normal pregnancies. In the second report by Hung et al. [5] examining 61 placentas in women without labor pains consisting of 14 with normal pregnancy (neither FGR nor PE), 14 with FGR alone, 18 with PE alone, and 15 with both FGR and PE [5], placental autophagy monitored by LC3B-II expression was not increased in women with PE

265 alone, but was increased in women with FGR irrespective of the presence or absence of PE compared to normal pregnancy [5]. As FGR was included in the criteria for diagnosis of severe PE in the study by Oh et al. [3], Hung et al. [5] considered that the difference in placental levels of LC3-II between PE and normal women reported by $\mathrm{Oh}$ et al. [3] was due to FGR rather than PE. However, placental LC3-II was significantly

270 increased in women with PE even in the absence of FGR compared to women with normal pregnancy in this study. Although the reason for these conflicting results is somewhat unclear, we propose the following explanation. The severity of PE differed between our study and that by Hung et al. [5]: the GW at delivery was $37.4 \pm 2.8$ for 18 women with PE alone and $35.2 \pm 3.4$ for 15 with both FGR and PE in the study by

275 Hung et al. [5], while the value was $34.6 \pm 2.9$ for 13 women with PE in this study; and women with hypertension and proteinuria $\geq 1+$ on dipstick test were included as PE in the study by Hung et al. [5]. As the dipstick test shows a false positive result more often than the protein-to-creatinine ratio $(\mathrm{mg} / \mathrm{mg}) \geq 0.27$ used in this study [17], there may have been women with no significant proteinuria among the 18 women with PE alone in 280 the study by Hung et al. [5].

In this study, although the number of cases of FGR was small and all FGR infants were born to PIH women, FGR placentas showed a significantly higher LC3-II level compared to control placentas (Fig. 4). In addition, the degree of fetal growth appeared to be inversely correlated with LC3-II level (Fig. 5, left panel). These results confirmed 285 the active autophagy in the placentas of FGR [5].

As approximately $15 \%-25 \%$ of women initially diagnosed with GH later develop PE $[18,19]$, GH has some features similar to PE. This may be true regarding autophagy; profiles of expression of both LC3-II and p62 resembled those of PE in this study, although differences in either level of LC3-II or p62 between the placentas of GH and

290 control women did not reach the level of significance, perhaps due to the limited number of the placentas tested (Fig. 2).

In conclusion, LC3-II level was significantly increased, while that of p62 was significantly reduced in the placentas of women with PIH compared to those of women with normal blood pressure irrespective of the presence or absence of FGR. LC3-II was also significantly increased in the placentas of women with PE irrespective of the presence or absence of FGR. These results suggested that autophagy is more active in the placenta in women with hypertensive disorders in pregnancy compared to those of normotensive pregnancies even in the absence of FGR. 
This study was supported by a Grant-in-Aid for Scientific Research from the Ministry of Education, Science, Sports, and Culture of Japan (No. 18791140 and No. 26462468), by grants from the Takeda Scientific Foundation, and by the JAOG Ogyaa Donation Foundation.

\section{Disclosure of interest}

The authors declare they have no financial conflicts of interest.

\section{Ethics approval}

This study was conducted after receiving approval from the ethics committee of Hokkaido University Graduate School of Medicine.

\section{Funding}

This study was not supported by a Grant-in-Aid for Scientific Research from the Ministry of Education, Science, Sports, and Culture of Japan (No. 18791140 and No. 26462468), by grants from the Takeda Scientific Foundation, and by the JAOG Ogyaa Donation Foundation. 


\section{References}

[1] Mizushima N, Ohsumi Y, Yoshimori T. Autophagosome formation in mammalian cells. Cell Struct Funct 2002; 27: 421-429.

[2] Lum JJ, DeBerardinis RJ, Thompson CB. Autophagy in metazoans: cell survival in the land of plenty. Nat Rev Mol Cell Biol 2005; 6: 439-448.

[3] Oh SY, Choi SJ, Kim KH, Cho EY, Kim JH, Roh CR. Autophagy-related proteins, LC3 and Beclin-1, in placentas from pregnancy complicated by preeclampsia. Reprod Sci 2008;15:912-920.

[4] Signorelli P, Avagliano L, Virgili E, Gagliostro V, Doi P, Braidotti P, Bulfamante GP, Ghidoni R, Marconi AM. Autophagy in term normal human placentas. Placenta. 2011;32:482-485.

[5] Hung TH, Chen SF, Lo LM, Li MJ, Yeh YL, Hsieh TT. Increased autophagy in placentas of intrauterine growth-restricted pregnancies. PLoS One. 2012;7:e40957.

[6] Hung TH, Hsieh TT, Chen SF, Li MJ, Yeh YL. Autophagy in the human placenta throughout gestation. PLoS One. 2013;8:e83475.

[7] Chang YL1, Wang TH, Chang SD, Chao AS, Hsieh PC, Wang CN. Increased autophagy in the placental territory of selective intrauterine growth-restricted monochorionic twins. Prenat Diagn. 2013;33:187-190.

[8] Curtis S1, Jones CJ, Garrod A, Hulme CH, Heazell AE. Identification of autophagic vacuoles and regulators of autophagy in villous trophoblast from normal term pregnancies and in fetal growth restriction. J Matern Fetal Neonatal Med. 2013;26:339-346.

[9] Avagliano L, Virgili E, Garò C, Quadrelli F, Doi P, Samaja M, Bulfamante GP, Marconi AM. Autophagy and human parturition: evaluation of LC3 expression in

340 placenta from spontaneous or medically induced onset of labor. Biomed Res Int. 2013;2013:689768.

[10] Avagliano L, Danti L, Doi P, Felis S, Guala M, Locatelli A, Maffeo I, Mecacci F, Plevani C, Simeone S, Bulfamante GP. Autophagy in placentas from acidotic newborns: an immunohistochemical study of LC3 expression. Placenta. 2013;34:1091-1094.

345 [11] Klionsky DJ, Abdalla FC, Abeliovich H, Abraham RT, Acevedo-Arozena A, Adeli $\mathrm{K}$, et al. Guidelines for the use and interpretation of assays for monitoring autophagy. Autophagy 2012; 8: 445-544.

[12] Tanida I, Ueno T, Kominami E. LC3 and autophagy. Methods Mol Biol 2008; 445 : $77-88$. 
350 [13] Bjørkøy G1, Lamark T, Pankiv S, Øvervatn A, Brech A, Johansen T. Monitoring atuophagic degradation of p62/SQSTM1. Methods Enzymol 2009; 452: 181-97.

[14] Itabashi K, Fujimura M, Kusuda S, Tamura M, Hayashi T, Takahashi T, et al. New normal birth-weight for Japanese infant according to gestational age at delivery. J Jpn Pediatr Soc 2010; 114: 1271-1293. (in Japanese)

355 [15] Jahania SM1, Sengstock D, Vaitkevicius P, Andres A, Ito BR, Gottlieb RA, Mentzer RM Jr. Activation of the homeostatic intracellular repair response during cardiac surgery. J Am Coll Surg. 2013;216:719-726.

[16] Huang J, Sun W, Huang H, Ye J, Pan W, Zhong Y, Cheng C, You X, Liu B, Xiong L, Liu S. miR-34a Modulates Angiotensin II-Induced Myocardial Hypertrophy by

360 Direct Inhibition of ATG9A Expression and Autophagic Activity. PLoS One. 2014;9:e94382.

[17] Yamada T, Kojima T, Akaishi R, Ishikawa S, Takeda M, Kawaguchi S, et al. Problems in methods for the detection of significant proteinuria in pregnancy. J Obstet Gynaecol Res 2014; 40: 161-6.

365 [18] Saudan P, Brown MA, Buddle ML, Jones M. Does gestational hypertension become pre-eclampsia? Br J Obstet Gynaecol 1998; 105: 1177-1184.

[19] Morikawa M, Yamada T, Yamada T, Cho K, Yamada T, Sakuragi N, Minakami H. Pregnancy outcome of women who developed proteinuria in the absence of hypertension after mid-gestation. J Perinat Med 2008; 36: 419-24. 
Akaishi R et al.,

Figure 1

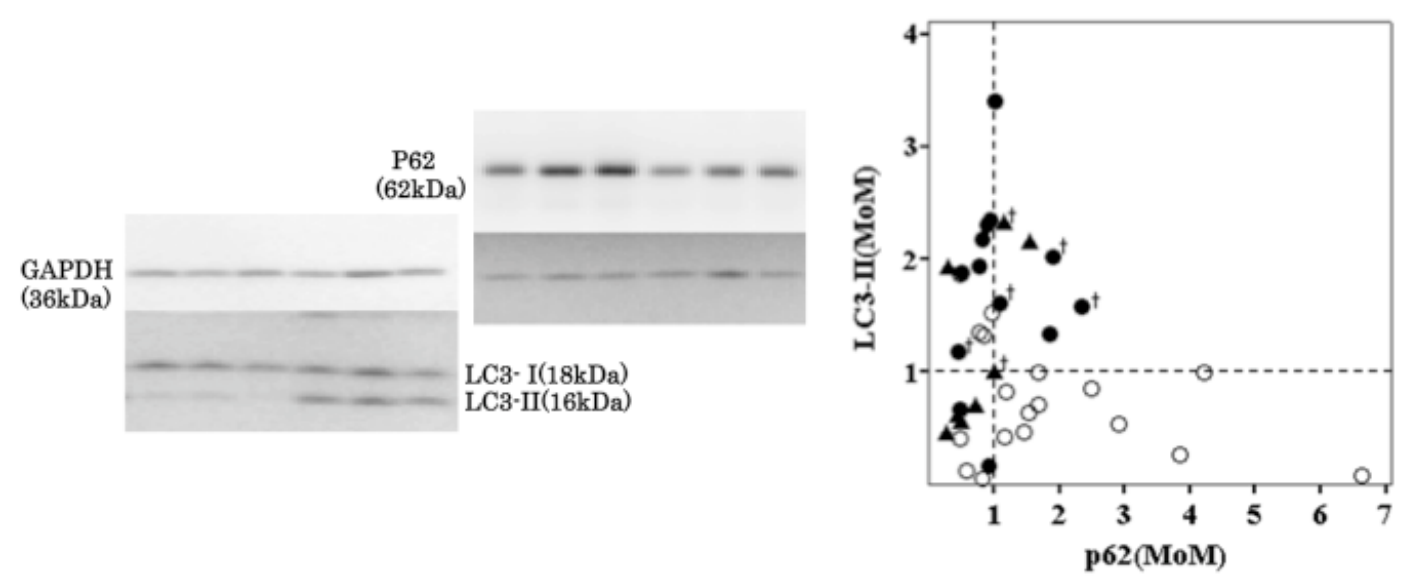


Akaishi R et al.,

$375 \quad$ Figure 2
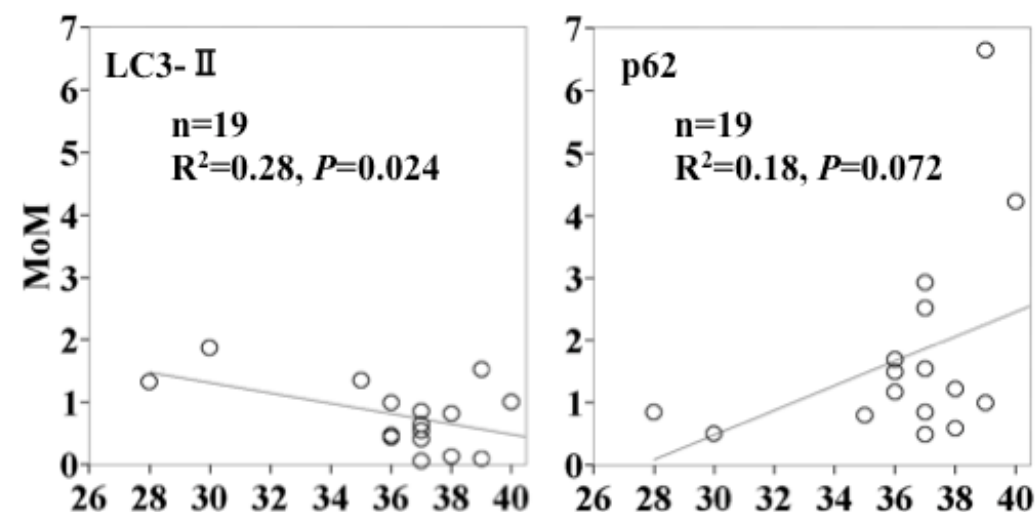

Control

- PE

$\triangle \mathrm{GH}$

$\dagger$ FGR
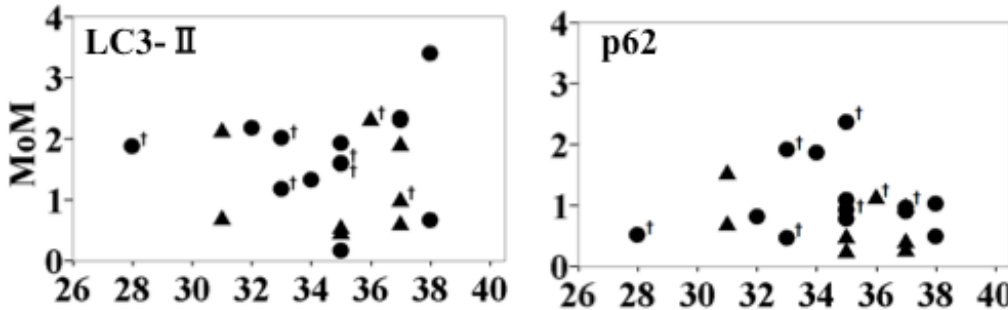

Gestational week at delivery 
Akaishi R et al.,

Figure 3

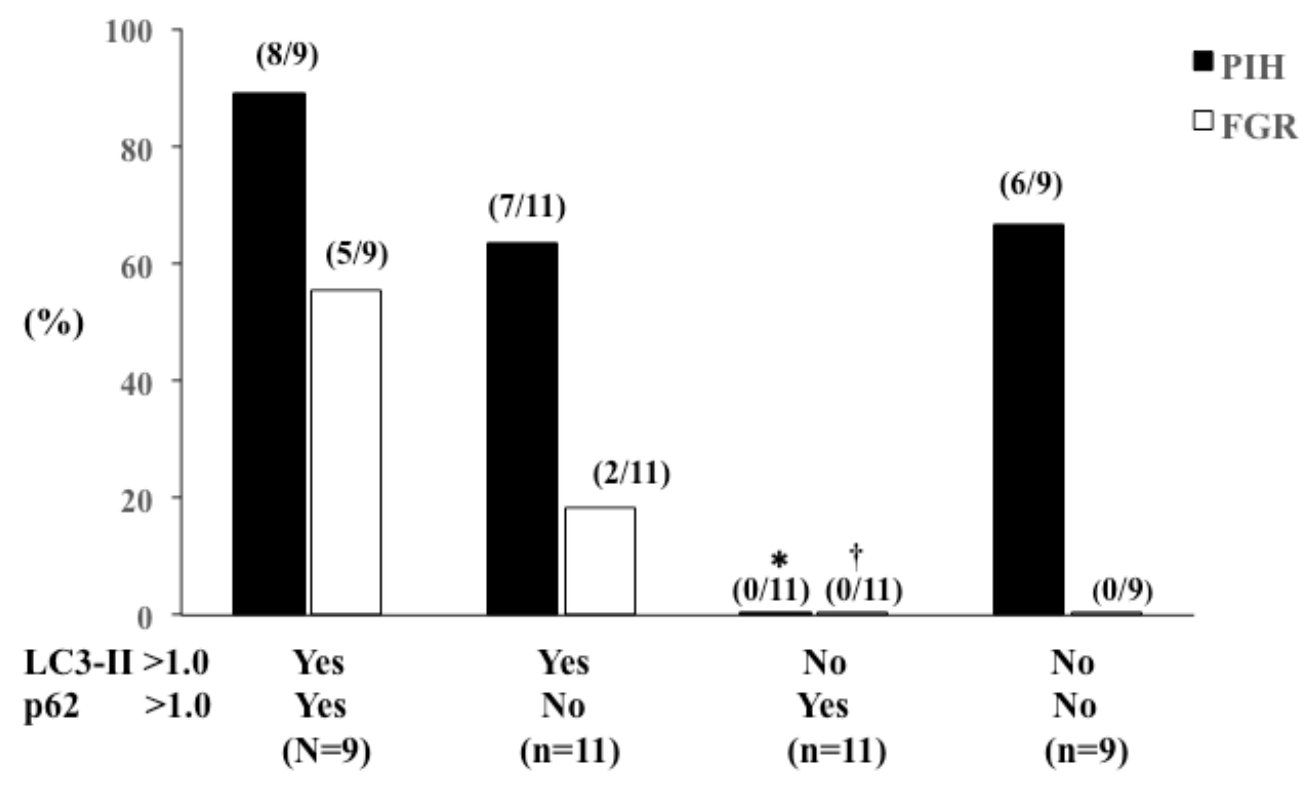


Akaishi R et al.,

Figure 4
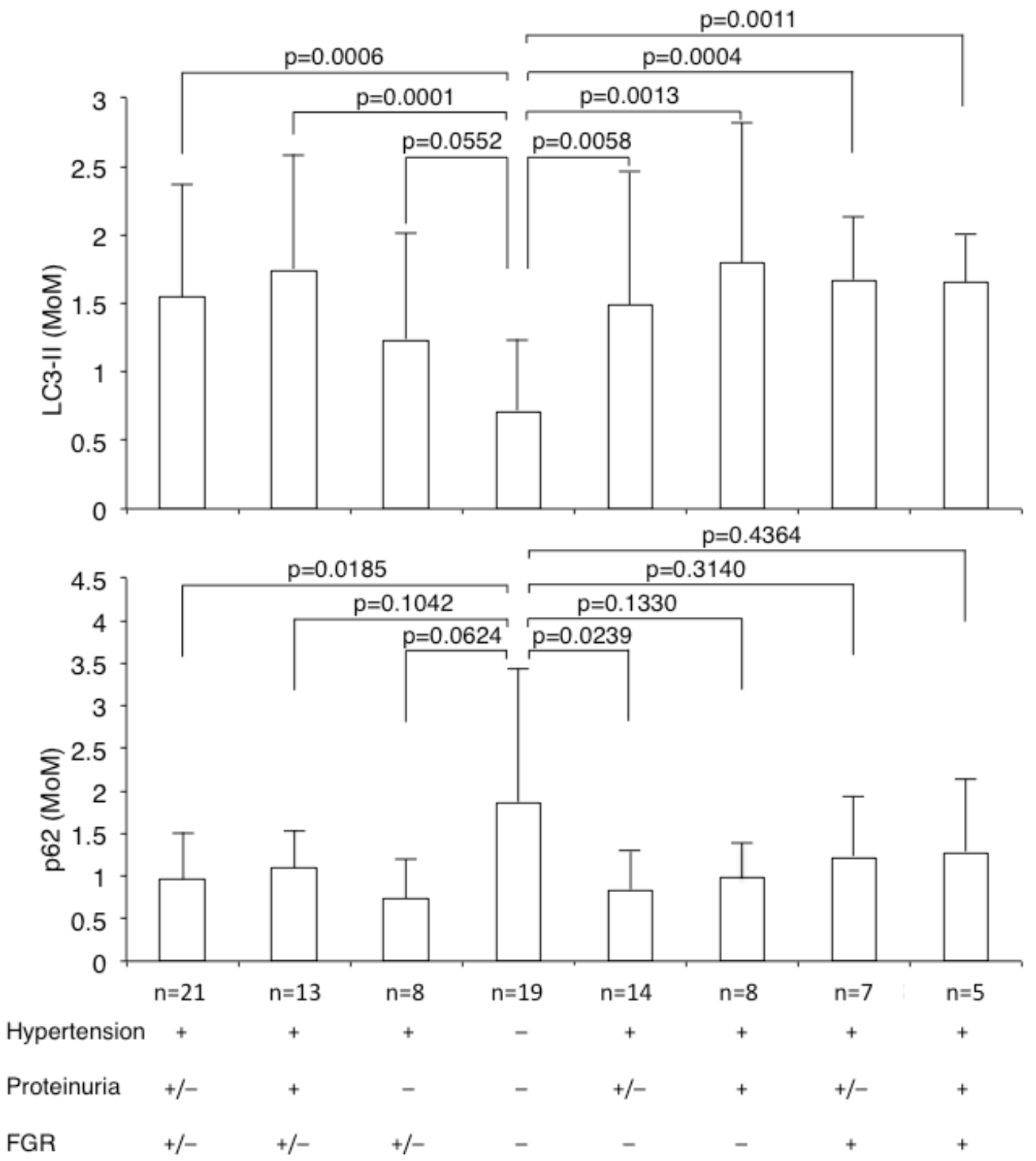
Akaishi R et al.,

Figure 5
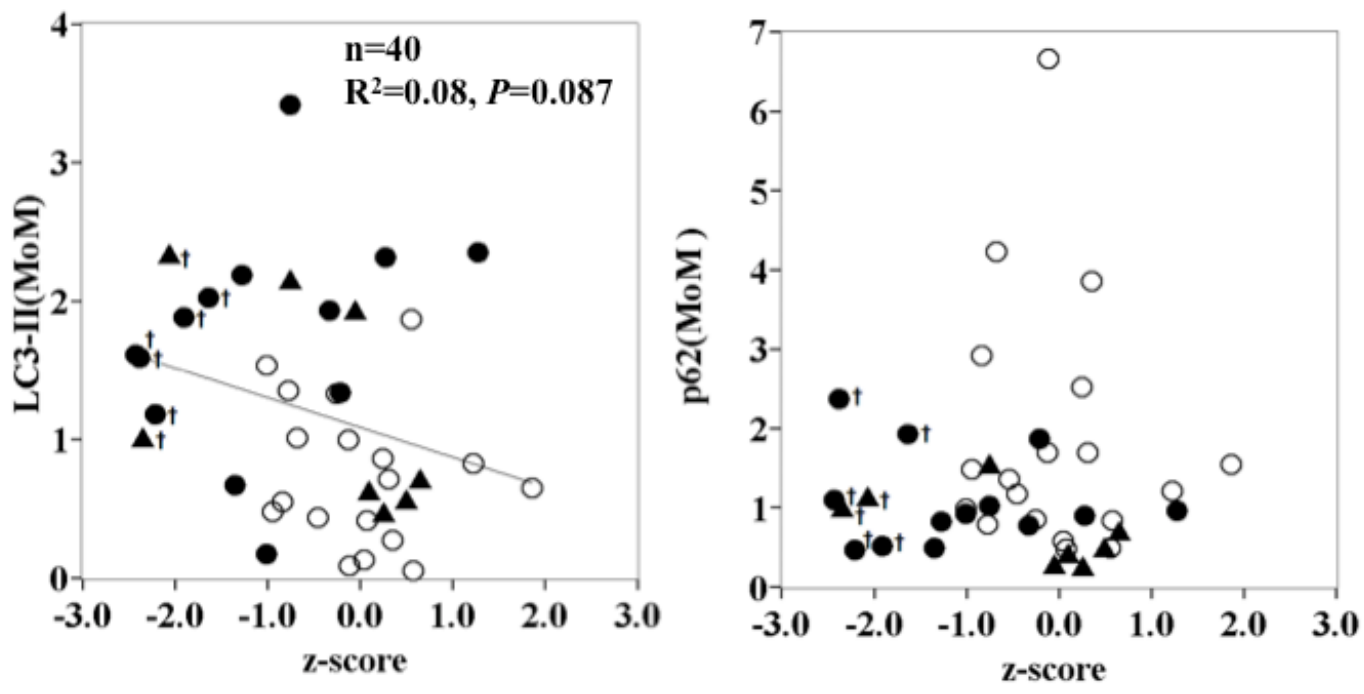

385 
Akaishi R et al.,

Figure 6
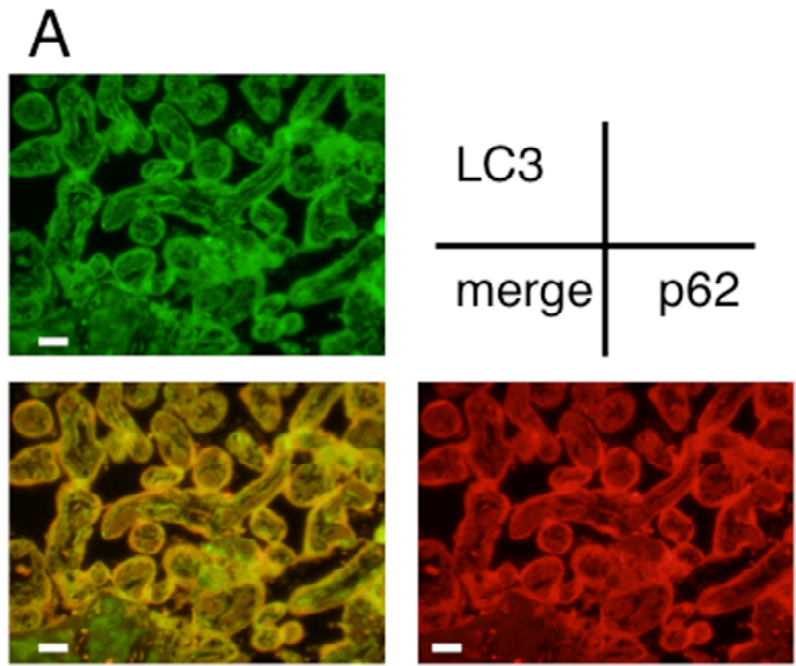

B
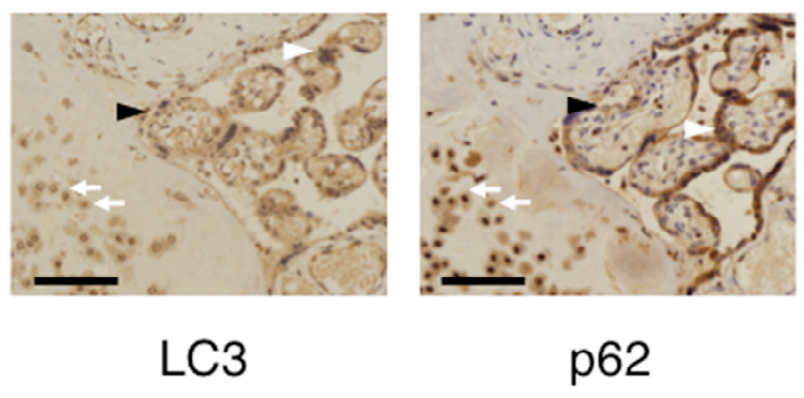
Table 1. Demographic characteristics of study subjects

\begin{tabular}{lllll}
\multicolumn{4}{c}{} & \multicolumn{3}{c}{ PIH } & \\
\cline { 2 - 4 } & PE & GH & Overall & Control \\
\hline No. of women & 13 & 8 & 21 & 19 \\
Maternal age (year) & $32.6 \pm 4.7$ & $34.6 \pm 6.0$ & $33.4 \pm 5.2$ & $30.5 \pm 5.2$ \\
Pre-pregnancy BMI $\left(\mathrm{kg} / \mathrm{m}^{2}\right)$ & $22.7 \pm 4.9$ & $22.9 \pm 5.5$ & $22.8 \pm 5.0$ & $21.3 \pm 2.8$ \\
GW at caesarean delivery & $34.6 \pm 2.9 *$ & $34.9 \pm 2.5$ & $34.7 \pm 2.5 *$ & $37.0 \pm 3$ \\
$\quad$ Preterm $(\mathrm{GW}<37)$ & $9(69 \%)$ & $5(63 \%)$ & $14(67 \%)$ & $6(32 \%)$ \\
Birth-weight $(\mathrm{kg})$ & $2.0 \pm 0.7^{*}$ & $2.2 \pm 0.5^{*}$ & $2.1 \pm 0.6^{*}$ & $2.7 \pm 0.6$ \\
$\quad$ z-Score & $-1.07 \pm 1.08^{*}$ & $-0.46 \pm 1.15$ & $-0.84 \pm 1.14^{*}$ & $-0.02 \pm 0.75$ \\
$\quad$ FGR & $5(38 \%)^{*}$ & $2(25 \%)^{*}$ & $7(33 \%)^{*}$ & $0(0.0 \%)$ \\
\hline
\end{tabular}

Data are presented as means \pm SD. BMI, body mass index; FGR, fetal growth restriction defined as a birth weight less than 10th percentile value for each gestational week at birth for Japanese infants; GH, gestational hypertension occurring at and after gestational week (GW) 20; PE, preeclampsia defined as condition with both gestational hypertension $(\mathrm{GH})$ and significant proteinuria $\left(>0.3 \mathrm{~g} /\right.$ day); ${ }^{*}, P<0.05$ versus Control. 
Table 2. LC3-II levels in two groups divided by GW at delivery

\begin{tabular}{|c|c|c|c|}
\hline GW at delivery & $<35$ & $>35$ & $P$-value \\
\hline Control (n=19) & $1.60 \pm 0.38(\mathrm{n}=2)$ & $0.65 \pm 0.43 *(n=17)$ & 0.052 \\
\hline $\mathrm{PIH}(\mathrm{n}=21)$ & $1.64 \pm 0.57(\mathrm{n}=7)$ & $1.50 \pm 0.94(n=14)$ & 0.689 \\
\hline $\operatorname{PE}(n=13)$ & $1.72 \pm 0.44(\mathrm{n}=5)$ & $1.76 \pm 1.01(\mathrm{n}=8)$ & 0.833 \\
\hline $\mathrm{GH}(\mathrm{n}=8)$ & $1.44 \pm 1.02(\mathrm{n}=2)$ & $1.16 \pm 0.79(\mathrm{n}=6)$ & 0.643 \\
\hline GW at delivery & $<37$ & $\geq 37$ & \\
\hline Control (n=19) & $1.08 \pm 0.55(\mathrm{n}=6)$ & $0.59 \pm 0.44 \dagger(n=13)$ & 0.102 \\
\hline $\mathrm{PIH}(\mathrm{n}=21)$ & $1.44 \pm 0.71(n=14)$ & $1.76 \pm 1.03(\mathrm{n}=7)$ & 0.400 \\
\hline $\operatorname{PE}(n=13)$ & $1.55 \pm 0.61(\mathrm{n}=9)$ & $2.19 \pm 1.13(\mathrm{n}=4)$ & 0.148 \\
\hline $\mathrm{GH}(\mathrm{n}=8)$ & $1.25 \pm 0.92(\mathrm{n}=5)$ & $1.20 \pm 0.67(\mathrm{n}=3)$ & 1.000 \\
\hline
\end{tabular}

Number in parenthesis indicates number of study subjects. GW, gestational week.

$\mathrm{PIH}$, pregnancy-induced hypertension including gestational hypertension $(\mathrm{GH})$ and pre-eclampsia (PE).

$*$ and $\uparrow, P=0.006$ and 0.010 vs. corresponding value of PIH group, respectively. 
Table 3. p62 levels in two groups divided by GW at delivery

\begin{tabular}{llll}
\hline GW at delivery & $<35$ & $\geq 35$ & $P$-value \\
\hline Control $(n=19)$ & $0.67 \pm 0.25(\mathrm{n}=2)$ & $2.00 \pm 1.61 *(\mathrm{n}=17)$ & 0.140 \\
PIH $(\mathrm{n}=21)$ & $1.12 \pm 0.64(\mathrm{n}=7)$ & $0.87 \pm 0.53(\mathrm{n}=14)$ & 0.535 \\
$\quad$ PE $(\mathrm{n}=13)$ & $1.12 \pm 0.72(\mathrm{n}=5)$ & $1.07 \pm 0.56(\mathrm{n}=8)$ & 0.724 \\
GH $(\mathrm{n}=8)$ & $1.14 \pm 0.60(\mathrm{n}=2)$ & $0.61 \pm 0.38(\mathrm{n}=6)$ & 0.286 \\
\multicolumn{1}{c}{ GW at delivery } & $<37$ & \multicolumn{2}{c}{$\geq 37$} \\
Control $(\mathrm{n}=19)$ & $1.08 \pm 0.45(\mathrm{n}=6)$ & $2.22 \pm 1.79 \dagger(\mathrm{n}=13)$ & 0.179 \\
PIH $(\mathrm{n}=21)$ & $1.07 \pm 0.63(\mathrm{n}=14)$ & $0.73 \pm 0.31(\mathrm{n}=7)$ & 0.322 \\
$\quad$ PE $(\mathrm{n}=13)$ & $1.20 \pm 0.45(\mathrm{n}=9)$ & $0.85 \pm 0.24(\mathrm{n}=4)$ & 0.710 \\
GH $(\mathrm{n}=8)$ & $0.84 \pm 0.52(\mathrm{n}=5)$ & $0.58 \pm 0.24(\mathrm{n}=3)$ & 0.571 \\
\hline
\end{tabular}

Number in parenthesis indicates number of study subjects. GW, gestational week.

$\mathrm{PIH}$, pregnancy-induced hypertension including gestational hypertension $(\mathrm{GH})$ and pre-eclampsia (PE).

$*$ and $\uparrow, P=0.004$ and 0.014 vs. corresponding value of PIH group, respectively. 